\title{
MoRfoANATOMÍA DE LAS FASES DE DESARROLLO DEL SICONO DE FICUS TUERCKHEIMII (Subg. SPHEROSUKE, SECT. Americanae, Moraceae)
}

\section{MORPHO-ANATOMICAL TRAITS OF THE SYCONE DEVELOPMENT PHASES OF FICUS tUERCKHEIMII (SUBg. SPHEROSUKE, SECT. AMERICANAE, MoraceAe)}

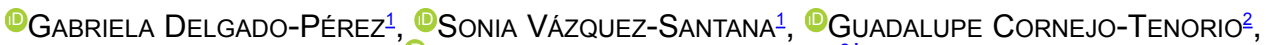 \\ (DUILLERMO IBARRA-MANRÍQUEZ $Z^{2 *}$
}

\begin{abstract}
${ }^{1}$ Laboratorio de Desarrollo en Plantas, Departamento de Biología Comparada. Facultad de Ciencias. Universidad Nacional Autónoma de México, Ciudad de México, México.

${ }^{2}$ Instituto de Investigaciones en Ecosistemas y Sustentabilidad. Universidad Nacional Autónoma de México, Morelia, Michoacán, México
\end{abstract}

*Autor de correspondencia: gibarra@iies.unam.mx

\begin{abstract}
Resumen
Antecedentes: El género Ficus incluye aproximadamente 850 especies, distribuidas en zonas tropicales de todo el mundo y para un bajo número de ellas, se ha descrito el desarrollo del sicono. El presente estudio aborda este tópico, caracterizando los cambios morfo-anatómicos del sicono de $F$. tuerckheimii.

Pregunta: ¿Las fases de desarrollo del sicono de F. tuerckheimii difieren en sus atributos morfo-anatómicos?

Especies de estudio: $F$. tuerckheimii (Moraceae).

Sitio y años de estudio: Veracruz, México (2018-2019).

Métodos: Se recolectaron siconos para describir la morfología, anatomía, color y tamaño en las diferentes fenofases de su desarrollo. Los siconos se fijaron para ser procesados e incluidos en paraplast y descritos mediante microscopía de luz o microscopía electrónica de barrido.

Resultados: Las fases de desarrollo difirieron en tamaño, así como en el color de la pared siconial y del ostíolo, particularmente en las fases más críticas de su interacción con polinizadores o dispersores (fases B, D y E). Las brácteas externas del ostíolo y el anillo ostiolar presentaron tricomoides; en la parte externa y basal del anillo ostiolar se registraron osmóforos. El registro de peines coxales y corbículas en la avispa hembra de Pegoscapus sp. indica que realiza una polinización activa.

Conclusiones: Las características morfoanatómicas de las fases de desarrollo del sicono en F. tuerckheimii son similares a las documentadas en otras especies del género. Sin embargo, existen diversos aspectos que deben explorarse con más detalle, con el objeto de determinar su similitud con otras poblaciones en las que se distribuye la especie en México y Centroamérica.
\end{abstract}

Palabras clave: Anillo ostiolar, brácteas ostiolares, Idarnes, osmóforos, Pegoscapus, tricomoides.

\begin{abstract}
Background: The genus Ficus includes about 850 species, found in tropical areas around the world. Nevertheless, studies on the syconium development has been carrying out only for a low number of these species. In the present study, morpho-anatomical changes along the phase development of the F. tuerckheimii syconium are described.

Question: Do the stages of the syconium development of $F$. tuerckheimii differ in their morpho-anatomical traits?

Study species: $F$. tuerckheimii (Moraceae).

Studied site and dates: Veracruz, Mexico (2018-2019).

Methods: Syconia were collected to describe their morphology, anatomy, color, and size during different phenophases. Fixed syconia at different development stages were processed and embedded in paraplast to be described by light microscopy or scanning electron microscopy. Results: Syconium development phases differed in size, as well as in the color of the syconia wall and the ostiole, particularly in the most critical phases of its interaction with pollinators or dispersers (phases B, D and E). The external bracts of the ostiole and the ostiolar ring presented trichomoids; ostiolar ring had osmophores. Combs and corbicles in the female wasp of Pegoscapus sp. indicate its active role as a pollinator of $F$. tuerckheimii.

Conclusions: Morpho-anatomical traits in the development syconium phases of $F$. tuerckheimii are similar to findings previously documented for other species of the genus. However, there are still several aspects that must be explored in more detail to determine their similarity in other populations where the species is found in Mexico and Central America.
\end{abstract}

Keywords: Idarnes, osmophores, ostiolar bracts, ostiolar ring, Pegoscapus, trichomoids.

Este artículo se encuentra bajo los términos de la licencia Creative Commons Attribution License CCBY-NC (4.0) internacional. 
La familia Moraceae comprende alrededor de 38 géneros y 1180 especies (Christenhusz \& Byng 2016), ubicadas en seis tribus, entre ellas Ficeae, con un único género, Ficus L., para el que se estiman más de 850 especies distribuidas en los trópicos de todo el mundo (Berg 1989, Berg \& Corner 2005, POWO 2020). Este diverso género se subdivide en los siguientes subgéneros (Pederneiras et al. 2015): i) Ficus, ii) Pharmacosycea (Miq.) Miq., iii) Spherosuke Raf. (antes Urostigma (Endl.) Miq.), iv) Sycomorus Raf., v) Synoecia (Miq.) Miq. y vi) Terega Raf. (antes Sycidium (Miq.) Mildbr. \& Burret). Únicamente los subgéneros Spherosuke y Pharmacosycea presentan especies en América, incluidas en las secciones Americanae (Miq.) Corner y Pharmacosycea (Miq.) Griseb., respectivamente (Pederneiras et al. 2015). Las especies de Ficus presentan formas de crecimiento variadas, desde árboles o arbustos hasta hemiepífitas y árboles estranguladores (Berg 1990, Berg \& Corner 2005, Clement \& Weiblen 2009). En el continente americano, se distribuyen desde el sureste de los Estados Unidos de Norteamérica (estado de Florida) hasta Argentina, en diversos tipos de comunidades vegetales y todas son monoicas (Ibarra-Manríquez et al. 2012).

Moraceae presenta una amplia variedad de inflorescencias (Clement \& Weiblen 2009); la de Ficus (denominada sicono o higo), es distintiva de los otros géneros de la familia, ya que consiste de un receptáculo carnoso en forma de urna, en cuyo interior se inserta un alto número de diminutas flores unisexuales. Avispas de la familia Agaonidae son las polinizadoras; para desempeñar esta función, entran al sicono a través de un orificio apical, cubierto de brácteas especializadas, conocido como ostíolo (Berg 1989, Ibarra-Manríquez \& Wendt 1992, Weiblen 2002, Cook \& Rasplus 2003, Datwyler \& Weiblen 2004, Berg \& Corner 2005). Finalizada la fecundación de las flores femeninas, se inicia el desarrollo de los frutos en el interior del sicono, ya sean drupas o aquenios (Berg \& Corner 2005), de manera que una vez que éstos maduran, el sicono funciona como la unidad de dispersión (IbarraManríquez \& Wendt 1992, Lomáscolo et al. 2010, IbarraManríquez et al. 2012).

El complejo desarrollo de inflorescencia a infrutescencia ha sido dividido en diversas fases de desarrollo, tomando en cuenta no sólo los cambios morfológicos del sicono sino, además, las intrínsecas relaciones con las avispas polinizadoras. Galil \& Eisikowitch (1968) fueron los primeros en abordar este tema, con base en las observaciones realizadas en la especie monoica $F$. sycomorus L. A las cinco fases propuestas en este ciclo (A-E), Smith \& Bronstein (1996) consideraron pertinente subdividir las fases A y C. Sintetizando estas contribuciones, las fases se reconocen de la manera siguiente: 1) fase A1 (pre-femenina), la cual comienza con el desarrollo del sicono, donde el ostíolo se encuentra cerrado debido a la superposición y compactación de las brácteas externas; el sicono crece rápidamente durante esta fase, 2) A2 (femenina), esta fase se distingue porque los estigmas de las flores femeninas se encuentran receptivos y los óvulos están maduros, 3) fase $\mathrm{B}$ (polinización), se reconoce por la entrada de la (s) avispa (s) femenina (s) cargadas de polen a través de las brácteas ostiolares; las avispas hembra polinizan las flores femeninas y se efectúa la fecundación; también ovipositan sus huevos en los ovarios de algunas de ellas (flores agalladas), 4) fase C1 (interfloral temprana), se reconoce por el inicio del desarrollo de las larvas que formarán la nueva generación de avispas (dentro de las agallas), y el inicio del desarrollo de los frutos que contienen a las semillas, 5) fase C2 (interfloral intermedia), en la que continúa desarrollándose la nueva generación de avispas y las semillas, y debido a ello, los frutos incrementan su tamaño y la cavidad dentro del sicono se reduce, 6) fase C3 (interfloral tardía), que se reconoce porque las avispas están bien desarrolladas y las semillas casi maduras, 7) fase D (estaminada), inicia con la dehiscencia de las anteras y la emergencia de las avispas macho de las agallas, que fecundan y liberan a las avispas hembras, quienes transportan polen y abandonan el sicono a través de un túnel hecho por los machos, en busca de siconos en fase B y 8) fase E (post-floral), reconocida por el aumento de tamaño, maduración de los frutos con semillas, y en la consistencia y/o color del sicono, lo que deriva en su consumo por frugívoros y su eventual dispersión. Estas fases de desarrollo del sicono son ilustradas y descritas con detalle por Piedra-Malagón et al. (2019) para la especie mexicana $F$. petiolaris Kunth. Cabe destacar que los siconos despliegan importantes contrastes respecto a su tamaño, texturas y colores al madurar, que los frugívoros detectan principalmente por el color o aroma que manifiestan (Lomáscolo et al. 2010). En general, la fructificación en Ficus es asincrónica, de manera que los frutos están disponibles durante la mayor parte del año (Korine et al. 2000, Machado et al. 2005), tienen un alto contenido de azúcares y son fuente de alimento para numerosos vertebrados (Shanahan et al. 2001, Lomáscolo et al. 2008, 2010).

Después del estudio pionero de Galil \& Eisikowitch (1968) en F. sycomorus, Newton \& Lomo (1979) caracterizaron el desarrollo del sicono de la especie monoica $F$. vogelii, presente en África e indicaron que las flores masculinas y femeninas no presentan ningún orden aparente, sin diferencias en tamaño o función de las flores femeninas; los autores identificaron también la principal avispa polinizadora. Baijnath \& Ramcharun (1983) aportaron información sobre el desarrollo del sicono de $F$. capensis Thunb. (África), con énfasis en el desarrollo de las flores y su relación con su polinizador. Verkerke (1986, 1987, 1988) destacó el acoplamiento entre las fases de 
desarrollo del sicono de tres especies del Viejo Mundo y sus avispas polinizadoras; en 1989, sintetizó estos hallazgos y aportó descripciones básicas de ciertas estructuras del sicono en tres especies americanas de la sección Pharmacosycea ( $F$. insipida Willd., F. maxima Mill. y F. yoponensis Desv.).

Baijnath \& Naicker (1989) abordaron el desarrollo del sicono de la especie africana $F$. ingens (Miq.) Miq., destacando que las fases de desarrollo propuestas por Galil \& Eisikowitch (1968), se distinguen claramente y describieron con detalle los tricomas de la epidermis, pared del receptáculo, ostíolo y flores. Hernández-Sosa \& Saralegui-Boza (2001) indicaron los cambios de tamaño y color, así como aspectos generales de las flores y avispas asociadas en las distintas fases de desarrollo de $F$. aurea Nutt., en la isla de Cuba. Berg \& Corner (2005) incluyeron información general del ostíolo y pared del sicono, flores y sus tricomas asociados, frutos, semillas y polen para diversas especies de la Flora Malesiana. Un año después, se publicó un estudio centrado en $F$. racemosa L. en China (Zhang et al. 2006), en la que las fases de desarrollo del sicono se relacionaron con el grosor de la pared y el diámetro durante distintas épocas del año; la tasa de desarrollo del sicono alcanzó su punto máximo durante la fase B. Un estudio particular sobre los tricomas y estructuras secretoras del sicono de F. enormis Mart. ex Miq., fue realizado por Machado et al. (2013). Fan et al. (2019) estudiaron la anatomía de la pared del sicono en 21 especies y proponen la existencia de cinco tipos de pared. Al mismo tiempo, Piedra-Malagón et al. (2019) describieron las distintas fases de desarrollo del sicono de $F$. petiolaris, ilustrando cada fase y su asociación con distintas especies de avispas, principalmente de sus polinizadoras y las parásitas de éstas.

El presente trabajo se enfoca en caracterizar las fases de desarrollo del sicono de F. tuerckheimii Standl., con el objetivo principal de aportar información en el entendimiento de la relación simbiótica del género Ficus con las avispas que las polinizan. Se pretende determinar si pueden encontrarse atributos morfológicos, sobre todo del exterior del sicono, que permitan identificar fases particularmente críticas para la interacción (Fases B y D). Una situación análoga sucedería cuando los siconos se encuentran maduros (Fase E), ya que una morfología distintiva facilitaría la realización de estudios relacionados con dispersores potenciales.

\section{Materiales y métodos}

Ficus tuerckheimii formó parte del complejo $F$. aurea (Berg 2007, Ibarra-Manríquez et al. 2012), pero HernándezEsquivel et al. (2020), con base en análisis cuantitativos del tamaño de la hoja y del sicono, la reconocen como una especie independiente, cuya distribución incluye México y Centroamérica. Se colectaron ramas con siconos en las diferentes fases de desarrollo en seis individuos de F. tuerckheimii, en junio del 2018, en dos localidades del estado de Veracruz (Coatepec y Xalapa), México. Se colectaron ejemplares botánicos (Eva María Piedra Malagón 612 al 619), en ambas localidades de estudio; un duplicado de cada número se depositará en el Herbario Nacional (MEXU). Para el establecimiento de las ocho fases típicas de desarrollo de los siconos, se utilizó la caracterización de Smith \& Bronstein (1996) y Piedra-Malagón et al. (2019). Las muestras se fijaron en FAA (formaldehído, etanol, ácido acético y agua destilada), fueron deshidratadas e incluidas en Paraplast, se obtuvieron cortes histológicos de entre 5-8 $\mu \mathrm{m}$ de grosor, con un micrótomo rotatorio American Optical 820 , se tiñeron con safranina y verde rápido o algunos se usaron para aplicar la prueba histoquímica de vainillina para detectar taninos; las laminillas seleccionadas se fotografiaron con un microscopio óptico Olympus Provis AX70, que tiene adaptada una cámara digital Evolution MP 5.1. Los siconos seccionados longitudinalmente fueron fotografiados en un microsopio estereoscópico Carl Zeiss. Además, se procesaron distintas estructuras del sicono fijadas en FAA o etanol al $70 \%$, las cuales se deshidrataron, se llevaron a punto crítico, se adhirieron a portamuestras metálicos y se cubrieron con oro para obtener fotomicrografías con un microscopio electrónico de barrido Jeol JSM-5310LV (Márquez-Guzmán et al. 2016). Un total de 30 siconos en cada etapa se midieron con un vernier y el programa Leica Application Suite Version 3.4.0, para determinar el largo (rango), ancho (rango), así como el promedio y desviación estándar de ambas medidas. El largo del sicono se midió desde su base hasta el ostíolo, mientras que el ancho se registró en una línea transversal al largo, en la parte media del sicono. Las diferencias en el tamaño de los siconos, entre las fases de desarrollo de los siconos, fueron evaluadas por medio de modelos lineales generalizados y comparaciones múltiples de medias (Prueba poshoc de Tukey), utilizando el paquete 'multcomp' (Hothorn et al. 2008) en R ( $\underline{\text { R Core Team 2018). }}$ ).

\section{Resultados}

Desarrollo de los siconos. Fase A1. Los siconos se encuentran en posición axilar a las hojas, generalmente en pares, aunque a veces alguno de ellos puede abortar; son sésiles, cada uno está cubierto totalmente por dos brácteas basales, las cuales están fusionadas en la base (Figuras 1A, 2A). Los siconos presentan una estructura discoide en su base, la cual persiste a lo largo de todas las fases (Figura 1A-L). Al emerger de las brácteas, los siconos son esféricos, verdes, con la superficie lisa, glabros, con un largo y ancho promedio de 8.9 y $9.2 \mathrm{~mm}$, respectivamente. 

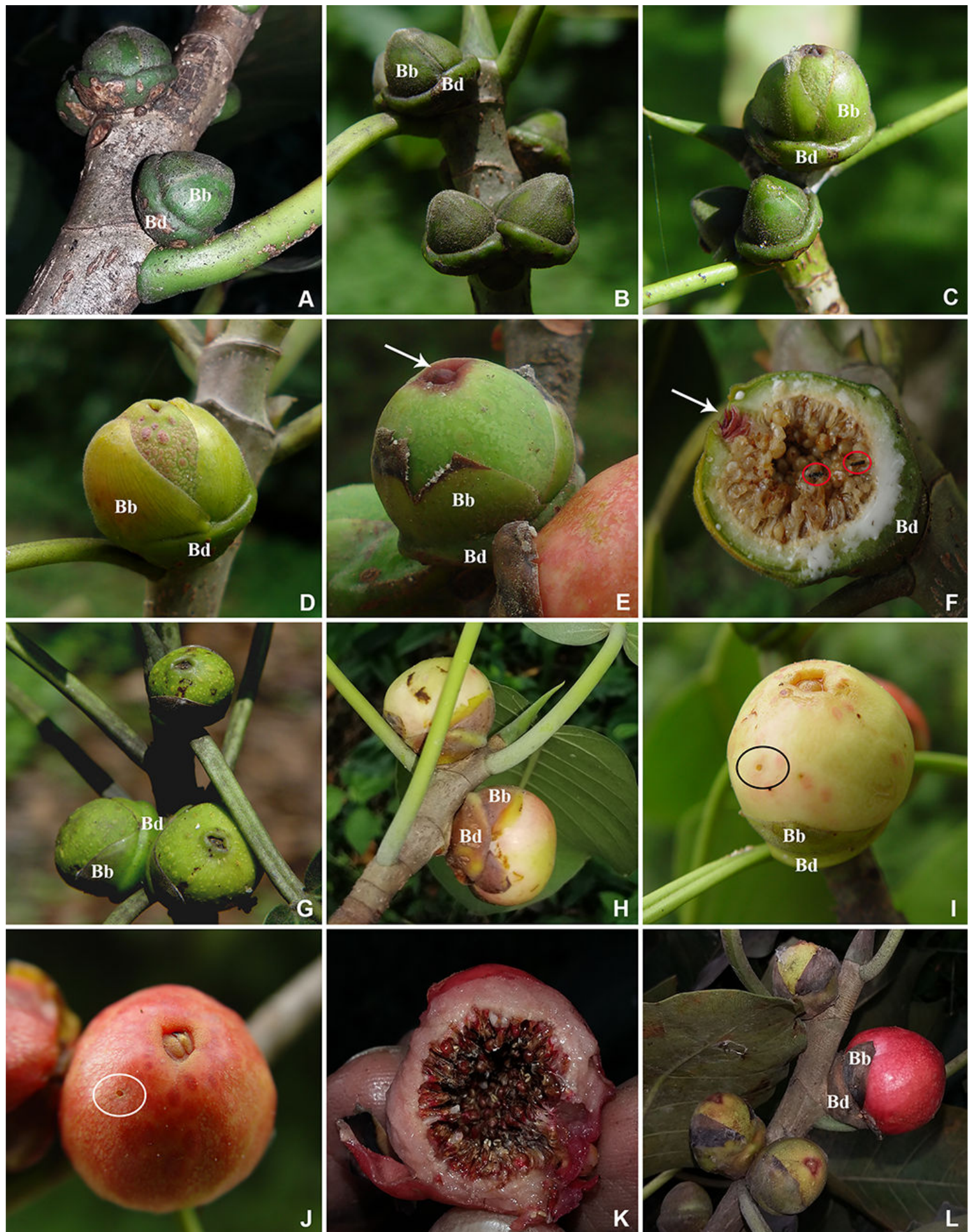

Figura 1. Fases de desarrollo del sicono de Ficus tuerckheimii. A) Fase A1. B) Fase A2. C) Fases A1 (sicono inferior) y B (sicono superior). D) Fase B. E) Detalle del ostíolo (área rojiza señalada con una flecha) durante la Fase B. F) Fase B. Corte longitudinal del sicono, en las que se observan dos avispas polinizadoras del género Pegoscapus (círculos rojos) y el ostíolo (flecha). G) Fase C1 (sicono superior), C2 (sicono lado izquierdo) y $C 3$ (sicono lado derecho). H) Fase D. I) Detalle de sicono en Fase D, pared del sicono con túnel (círculo). J) Fase E. En la pared del sicono se observa el orificio (círculo) cavado por las avispas macho y por el que emergieron las avispas hembra. K) Fase E, corte transversal del sicono. L) Siconos en distintas fases de desarrollo ( $A 2$, sicono lado inferior izquierdo; $B$, sicono en la parte superior y los dos siconos de la parte media de la ramilla; $E$, sicono lado superior derecho). Bb (brácteas basales), Bd (base discoide). 
En el ápice del sicono se localiza el ostiolo, conformado por brácteas ostiolares, con un borde formado por la parte apical de la pared (el anillo ostiolar) (Figura 2B-C). Las brácteas se dividen en: i) externas (o superficiales), ii) transicionales, ubicadas en la parte media del ostiolo y entrelazadas horizontalmente, y iii) internas o de la pared, con un arreglo inclinado hacia el interior de la cavidad del sicono (Figura 2A-B). Las brácteas externas o superficiales tienen mayor grosor con respecto a las demás y en su epidermis abaxial, que es la que está en contacto con el exterior, se observan células epidérmicas elongadas, formando varios estratos con aspecto en empalizada, llamadas tricomoides, de naturaleza secretora (Figura 2E); el mesófilo de estas brácteas está conformado por parénquima de células isodiamétricas, la epidermis adaxial es uniestratificada y presenta células taníferas (Figura 2F-G). Las células taníferas fueron detectadas por la coloración pardo-rojiza de los taninos condensados en la pared del sicono (datos no mostrados). Las brácteas transicionales se encuentran paralelas a las externas, son lanceoladas, delimitan pequeños espacios entre ellas, con un mesófilo parenquimático sin espacios intercelulares aparentes; su epidermis abaxial y la adaxial generalmente carecen de taninos. Las brácteas internas guardan un mayor contacto con la pared interna del receptáculo, no se entrelazan entre sí y tienen sus extremos libres, péndulos y verticales hacia el interior del sicono, mientras que sus epidermis carecen de taninos y el mesófilo está constituido por células parenquimáticas (Figura 2B, E). La pared del sicono es gruesa y en su interior se encuentran múltiples primordios florales que se intercalan, sin un orden aparente; los femeninos darán lugar a flores sésiles o pediceladas (Figura 2D). Los primordios masculinos se presentan en menor proporción que los femeninos. Cada botón floral se encuentra protegido por una bractéola que sobrepasa el tamaño de los botones florales y cubre a los sépalos (Figura 2D).

Fase A2. La caracterización morfológica descrita en la primera fase es similar, con un incremento de tamaño del sicono (en promedio, $10.7 \mathrm{~mm}$ de largo y $9.5 \mathrm{~mm}$ de ancho); el ostiolo está hundido con respecto al anillo ostiolar (Figuras 1B, 2H-J). Las brácteas de transición presentan características similares a las observadas en la Fase A1, en tanto las internas son ligeramente laxas, separadas unas de otras y sus extremos libres se extienden hacia el interior del sicono (Figura 2H-I); los tricomoides se registran en la superficie de las brácteas ostiolares externas y en el anillo ostiolar (Figura 2I-J, L). La pared que forma el receptáculo de la inflorescencia presenta múltiples estratos parenquimáticos, con algunas células taníferas dispersas (Figura 2L). Las flores femeninas maduran y los estigmas están receptivos formando un sinestigma (Figura $2 \mathrm{H}, \mathrm{K}$ ).
Fase B. Continúa el crecimiento del sicono $(12.1 \mathrm{~mm}$ de largo $\times 11.1 \mathrm{~mm}$ de ancho, en promedio), el cual adquiere una tonalidad verde pálido, con máculas verde amarillentas a ligeramente rojizas y, a partir de esta fase, las brácteas basales lo cubrirán sólo parcialmente (Figura 1C-E). Esta fase se caracteriza por la entrada de las avispas al sicono (Figuras 1F, 2M-O), que polinizarán las flores femeninas (Figura 2Q), lo que se asocia con el color rojizo que presenta el anillo ostiolar y las brácteas del ostíolo (Figura 1E-F). Las brácteas externas presentan abundantes tricomoides, que forman casi la mitad de su grosor e incluso se extienden hacia la parte interna y externa del anillo ostiolar (Figura 2O-P), en tanto que las brácteas de transición e internas se notan menos compactas (Figura 2P). En la base y parte externa del anillo ostiolar se registran osmóforos con estomas (Figura 2R-S).

Fase C1. Al inicio de esta fase el ostíolo y el anillo ostiolar se tornan verdes con máculas verde amarillentas (Figura 1G), mientras que el sicono aumenta nuevamente de tamaño con respecto a la fase previa, con un promedio de largo y ancho de 13.9 y $13.5 \mathrm{~mm}$, respectivamente. El ostiolo permanece ligeramente cóncavo (Figura 3A-C). Las brácteas externas pierden su rigidez, principalmente las más externas y los tricomoides siguen siendo evidentes, pero van colapsándose (Figura $3 \mathrm{H}$ ). Las brácteas de transición presentan una disposición más curva, con espacios pequeños entre sí, las brácteas internas más delgadas, descendentes, firmes entre ellas, debido al desarrollo de los frutos y a las agallas que contienen a las larvas de las avispas; las polinizadoras se encuentran muertas en la cavidad siconial (Figura 3A, D). Los osmóforos se distinguen como áreas abultadas, secretoras y con estomas abiertos (Figura 3E-G).

Fase C2. El sicono tiene una apariencia similar a la de la fase anterior (Figura 1G), pero se registró una disminución de sus dimensiones (promedio de $11.2 \mathrm{~mm}$ en su largo y ancho). Las brácteas ostiolares intermedias se ven más compactas; el ostiolo se observa ligeramente plano a cóncavo (Figura 3I-K) y los tricomoides de las brácteas externas se reducen (Figura 3M). Las agallas con larvas de avispas (ovarios con tonalidad negra) y los frutos (pardos pálidos) continúan su desarrollo en el interior del sicono, lo que conlleva a una evidente reducción de la cavidad siconial (Figura 3I, L).

Fase C3. Nuevamente la apariencia del sicono es similar al observado en la Fase C2 (Figura 1G), pero su tamaño promedio es mayor al de las dos fases previas $(16.8 \mathrm{~mm}$ tanto en largo como en ancho). El anillo ostiolar delimita al ostiolo, de manera que éste ocupa ahora una posición más profunda (Figuras 1G, 3N, Q-R); los tricomoides de las brácteas externas reducen su tamaño y se desintegran y éstas se separan ligeramente unas de otras y tienen un 

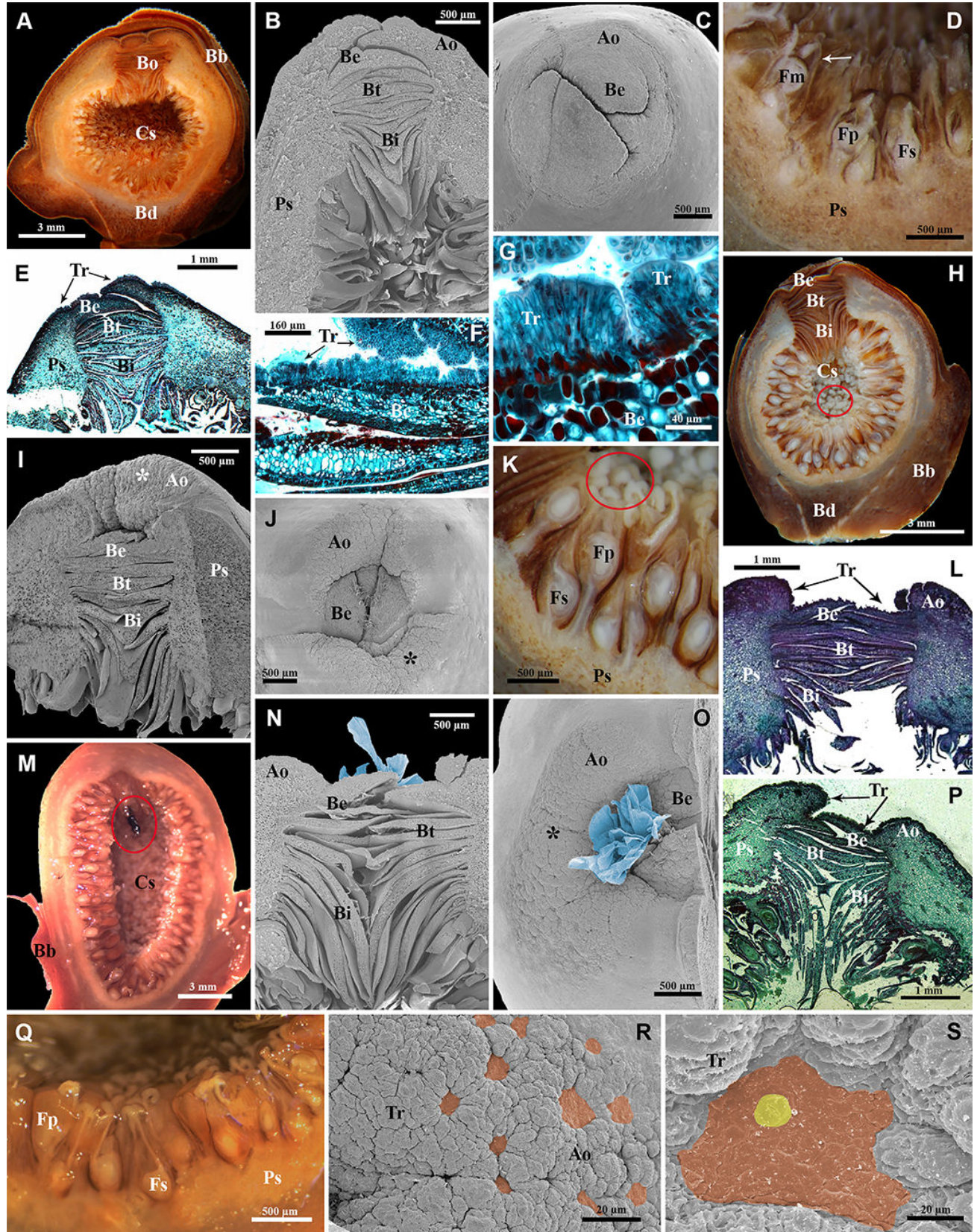

Figura 2. Morfoanatomía de los siconos de Ficus tuerckheimii en las fases de desarrollo A1-B. Fase A1. A-B) El corte longitudinal del sicono permite observar la cavidad siconial, el ostíolo y las brácteas basales y ostiolares; dentro de estas últimas, las externas son más engrosadas que las transicionales, que presentan una disposición más horizontal, en tanto que las internas se dirigen hacia la cavidad siconial. C) Brácteas ostiolares externas y anillo ostiolar. D) Flores inmaduras cubiertas por brácteolas (flecha) y sépalos. E) Brácteas ostiolares, la epidermis de las externas tiene tricomoides al igual que el anillo ostiolar. F-G) Detalle de los tricomoides alargados y pluricelulares en la epidermis abaxial de las brácteas externas; también hay células taníferas (con contenido rojo). Fase A2. H) Corte longitudinal del sicono mostrando la cavidad siconial en la que los estilos de las flores femeninas están más desarrollados, con los estigmas unidos para formar el sinestigma (círculo). I) Detalle del ostíolo mostrando el anillo ostiolar con tricomoides (asterisco)y la diferenciación entre los tres tipos de brácteas ostiolares. J) Vista frontal de las brácteas ostiolares externas con el anillo ostiolar evidente y tricomoides (asterisco). K) Flores femeninas pediceladas y sésiles formando un sinestigma (círculo). L) Brácteas ostiolares externas y parte interna del anillo ostiolar con tricomoides en su epidermis. Fase B. M) Avispa polinizadora (Pegoscapus sp.) (círculo) entrando a la cavidad siconial. N) Los tres tipos de brácteas ostiolares se delimitan claramente; en la entrada del ostíolo se destacan restos de las alas de las avispas (áreas en azul). O) Acercamiento al anillo ostiolar y las brácteas ostiolares externas con tricomoides (se observan como estructuras rugosas, se destacan con un asterisco); entre las brácteas ostiolares externas quedaron atrapadas las alas de las avispas hembra (estructuras en azul) de Pegoscapus sp., algunas de ellas tocando áreas del anillo ostiolar. P) Acercamiento al reborde del anillo ostiolar y a las brácteas ostiolares externas; en éstas y en el anillo ostiolar se destacan los tricomoides. Q) Flores femeninas con diferente longitud de los estilos. R-S) Parte externa del anillo ostiolar con tricomoides (superficies rugosas grises) y osmóforos (áreas naranjas); en color amarillo, se observa un estoma. Ao (anillo ostiolar), Bb (brácteas basales), Bd (base discoide), Be (brácteas externas), Bi (brácteas internas), Bo (brácteas ostiolares), Bt (brácteas transicionales), Cs (cavidad siconial), Fm (flor masculina), Fp (flor femenina pedicelada), Fs (flor femenina sésil), Ps (pared del sicono), Tr (tricomoides). 

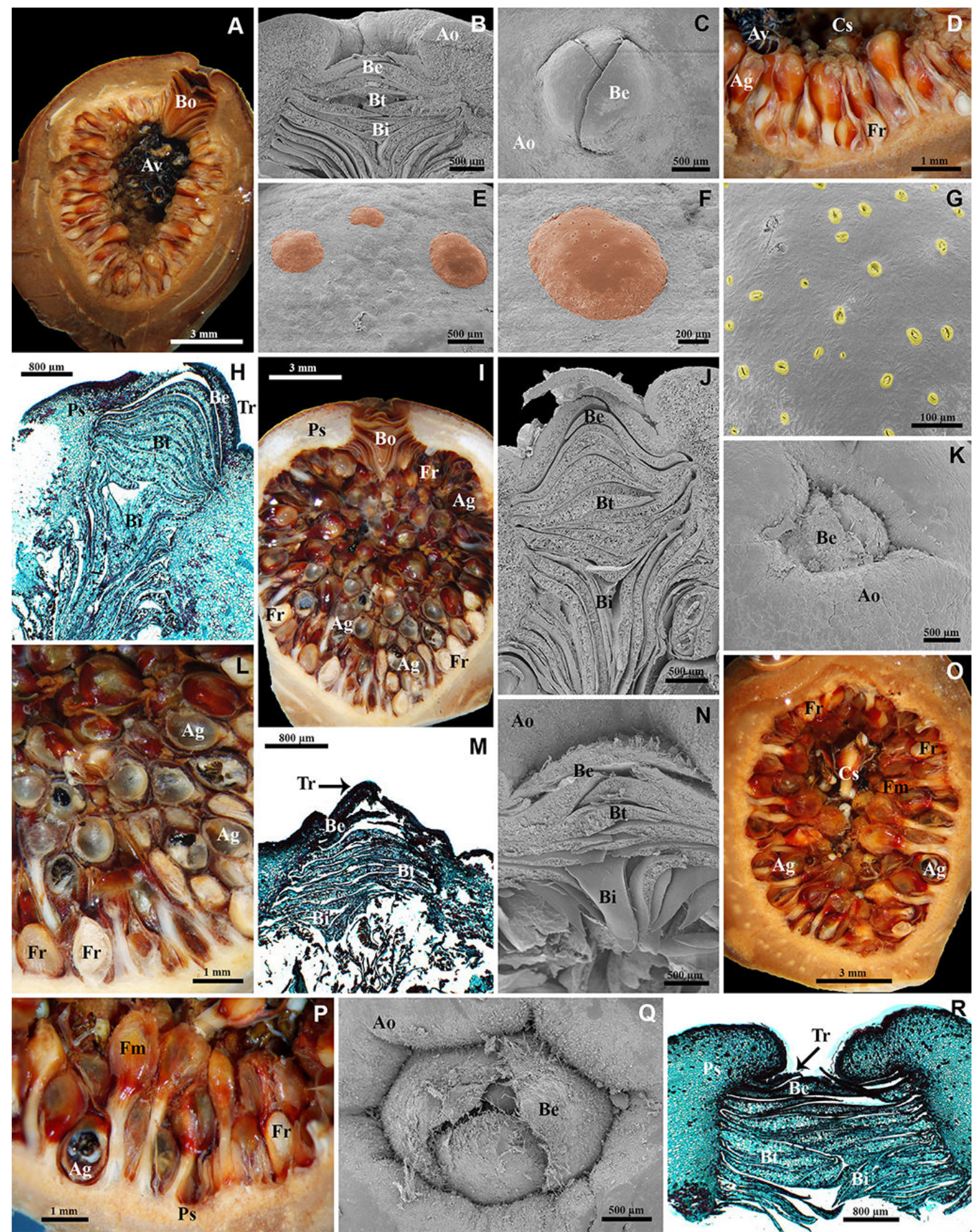

Figura 3. Morfología de los siconos de Ficus tuerckheimii en las fases de desarrollo C1-C3. Fase C1. A-B) Corte longitudinal del sicono mostrando la cavidad siconial, con un arreglo de las brácteas ostiolares similar al de la Fase B; en A se observan varios individuos de Pegoscapus sp. C) Vista frontal de las brácteas ostiolares externas y parte del anillo ostiolar. D) Vista de la estructura interna del sicono que muestra el inicio del desarrollo de los frutos y agallas. E-F) Osmóforos (en naranja) de diferentes tamaños posicionados sobre la parte externa y basal del anillo ostiolar. G) Detalle de los osmóforos para destacar los estomas abiertos (en amarillo). H) Brácteas ostiolares externas en las que se observan los tricomoides en su epidermis. Fase C2. I, L) Corte longitudinal del sicono donde se observan los frutos más desarrollados (pardo-pálidos) y las agallas con avispas (tonos negros), con una reducción del tamaño de la cavidad siconial. J-K, M) Distintas vistas de las brácteas ostiolares; en el corte histológico de éstas se observan los tricomoides. Fase C3. N) Detalle de las brácteas ostiolares; las intermedias y externas presentan una disposición compacta entre ellas. O-P) Vista de la cavidad siconial ocupada por el desarrollo de los frutos y las avispas, que se distinguen entre sí, nítidamente. Q-R) Detalle del ostíolo que se encuentra más hundido, delimitado por el anillo ostiolar más elevado; en R las brácteas ostiolares y el anillo ostiolar presentan numerosas células taníferas. Ag (agalla que encierra avispas en desarrollo), Ao (anillo ostiolar), Av (avispas hembras Pegoscapus sp.), Be (brácteas externas), Bi (brácteas internas), Bo (brácteas ostiolares), Bt (brácteas transicionales), Cs (cavidad siconial), Fm (flor masculina), Fr (fruto), Ps (pared del sicono), Tr (tricomoides). 
menor diámetro que las brácteas de transición, en tanto que las internas, se observan laxas (Figura 3R). Las flores masculinas completan casi su desarrollo, al igual que los frutos y las avispas; la cavidad siconial casi desaparece (Figura 3O-P).

Fase D. Los siconos en esta etapa se tornan amarillentos (Figura 1H-I) y son tenuemente blandos al tacto; su tamaño promedio es de $16.4 \mathrm{~mm}$ de largo y $17.2 \mathrm{~mm}$ de ancho; la apariencia del ostíolo es similar a la fase previamente descrita (Figuras 1G, 4B-C). Internamente, las anteras de las flores masculinas están maduras (Figura 4A). Las brácteas externas están conformadas por células de parénquima, con tricomoides secretores (Figura 4C-D); las de transición e internas presentan una disposición irregular, con mayores espacios entre ellas (Figura 4B, D) y los osmóforos se encuentran colapsados (Figura 4E). Las avispas macho cavan túneles en la pared del sicono (Figura 1I), que permite que salgan las avispas hembra fecundadas y con polen.

Fase E. Los siconos son rojizos, tanto en la pared externa e interna, muy blandos al tacto, con un sabor ligeramente dulce y su tamaño promedio, cuando se compara con todas las fases previas, es el mayor $(20.9 \times 22.9 \mathrm{~mm}$ de largo y ancho, respectivamente); se observan orificios en el sicono (Figura 1J), que es la evidencia externa de los túneles por los que escaparon las avispas hembra; la cavidad siconial es amplia (Figura 4F). El ostiolo mantiene una disposición cóncava (Figura 4H); las brácteas externas e intermedias adquieren una disposición ordenada y muy juntas entre sí, en tanto que las internas se separan, presentando una apariencia más laxa (Figura 4G, I). Las flores femeninas y masculinas están senescentes, se observan agallas huecas, sin avispas dentro de ellas y las semillas se encuentran maduras en el interior de los frutos (Figuras $1 \mathrm{~K}, \underline{4 \mathrm{~F}}$ ).

Análisis del tamaño de los siconos. Con excepción de la Fase C2, el largo y ancho del sicono mostró un incremento gradual. Con respecto a la primera de estas dimensiones, las etapas de desarrollo mostraron diferencias estadísticamente significativas $\left(F_{7,224}=1,417, P<0.000\right)$. La Fase E fue la que presentó la diferencia más notable (Figura 5A). Por su parte, la comparación considerando el ancho de los siconos, también fue significativa $\left(F_{7,224}=1,247, P<0.000\right) \mathrm{y}$ coincide con el primer análisis respecto a la distintividad de la Fase E, aunque el ancho del sicono también es contrastante para otras fases ( $\underline{\text { Figura 5B }}$ ).

Avispas polinizadoras y cleptoparásitas. Se recolectaron durante las fases de desarrollo B y D del sicono las avispas polinizadoras de ambos sexos del género Pegoscapus sp. Las hembras son aladas (Figura 6A-B), con lamelas en las mandíbulas (Figura 6C). Las avispas presentan corbículas en el mesosoma (cavidades para el transporte de polen), peines coxales (Figura 6E-F) y un ovipositor (Figura 6A-B, D). Por su parte, los machos presentan una morfología contrastante, ya que son ápteros, ciegos y no tienen estructuras para transportar polen (Figura 6G-H $)$. En la fase $\mathrm{D}$, también se identificó la presencia de avispas hembras no polinizadoras (Idarnes sp.), que se caracterizan porque tienen ojos rojos y su ovipositor es más largo (igura 6I) del que presentan las avispas polinizadoras; la dimensión del ovipositor le permite atravesar la pared del sicono desde el exterior y ovipositar flores femeninas durante la fase B. En esta misma etapa, se encontraron avispas macho de Idarnes sp., que al igual que los de su sexo en Pegoscapus sp., carecen de alas y visión (Figura 6J-K).

\section{Discusión}

Desarrollo de los siconos. Las diferencias morfológicas externas más evidentes a lo largo de las fases de desarrollo del sicono de $F$. tuerckheimii, son el incremento gradual de su tamaño, que difiere de manera significativa entre ellas, así como los cambios de color de la pared siconial y del ostíolo (Figuras 1 y $\underline{5}$ ). Lo anterior concuerda, en lo general, con lo documentado para $F$. aurea (Hernández-Sosa \& Saralegui-Boza 2001) y en menor medida con $F$. petiolaris (Piedra-Malagón et al. 2019). Las diferencias entre esta última especie y $F$. tuerckheimii es que la pared externa del sicono de $F$. petiolaris presenta tricomas y que, en la Fase E, despliega una coloración verde amarillenta. Los únicos tricomas externos encontrados en la especie incluida en el presente estudio corresponden a los tricomoides, tanto en las brácteas externas del ostíolo, como en el anillo ostiolar (Figuras 2-4).

Los siconos muestran una morfología externa similar cuando inician su desarrollo (fases A1 y A2), con incrementos de tamaño no estadísticamente significativos (Figura 5), si bien internamente se presentan cambios importantes en las brácteas ostiolares y en la maduración de las flores pistiladas (Figura 2). Durante la Fase A1 se destacó una estructura discoide en la base de los siconos, más evidente en la medida que éstos se desarrollan (Figura 1). Esta estructura es característica del complejo $F$. aurea, ya que no se ha observado en otras especies de la sect. Americanae y permite el desarrollo del pedúnculo de los siconos (Hernández-Esquivel et al. 2020). No fue posible esclarecer el origen anatómico de esta base discoide, lo que demanda un estudio detallado. La inclusión de otras especies del complejo $F$. aurea aportaría información valiosa sobre la posible relevancia sistemática de esta estructura.

La Fase B es reconocida, con mayor facilidad, por la presencia de las avispas polinizadoras en el interior del sicono, un evento que pudo ser inferido por el registro de alas atrapadas en las brácteas ostiolares externas 

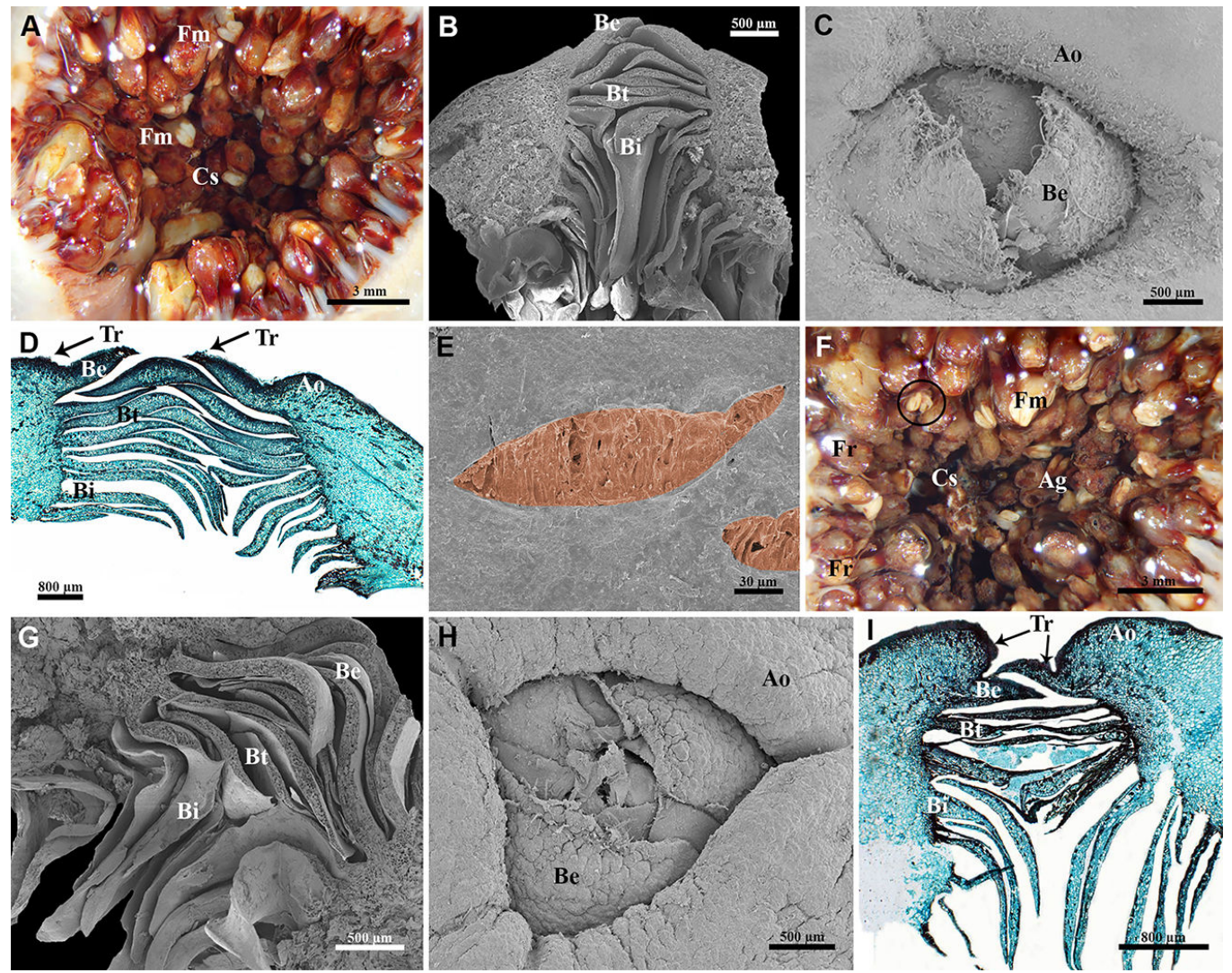

Figura 4. Morfoanatomía de los siconos de Ficus tuerckheimii en las fases de desarrollo D-E. Fase D. A) Las flores masculinas maduras (anteras blanquecinas), se encuentran dispersas en la cavidad siconial. B) Brácteas ostiolares externas e intermedias con disposición distinta a la que muestran las interiores. C) Detalle de las brácteas ostiolares externas y anillo ostiolar con tricomoides secretores. D) Brácteas ostiolares laxas. E) Osmóforos colapsados (en naranja). Fase E. F) Cavidad siconial con orificios de salida de las avispas en las flores agalladas, frutos y anteras (círculo). G) Disposición más laxa de las brácteas ostiolares, similares a la de la fase previa. H-I) Brácteas externas y anillo ostiolar con tricomoides colapsados. Ag (agalla con orficio por el que emergieron avispas), Ao (anillo ostiolar), Be (brácteas externas), Bi (brácteas internas), Bt (brácteas transicionales), Cs (cavidad siconial), Fm (flor masculina), Fr (fruto), $\operatorname{Tr}$ (tricomoides).

(Figura 2N-O), y también, directamente, al observarlas entre las últimas brácteas ostiolares internas, a punto de entrar hacia la cámara siconial (Figura 2M). Además, la observación de distintos siconos en fase $\mathrm{B}$, indica la presencia de 2 a 3 avispas hembra de Pegoscapus sp. en su interior (Figura 1F), lo que concuerda con lo registrado para otras especies (Bronstein \& Hossaert-McKey 1995, Gibernau et al. 1996, Hernández-Sosa \& Saralegui-Boza 2001, Piedra-Malagón et al. 2019), aunque en uno de los siconos se observaron más hembras (Figura 3A). Hasta donde se conoce, el color rojizo del ostíolo y del anillo ostiolar en esta fase de desarrollo (Figura 1E-F), que permite su contraste del resto de la pared siconial, no ha sido destacado previamente para otras especies del género.

La forma del ostíolo se ha relacionado con la morfología de la avispa polinizadora. Ramírez (1974) y Verkerke (1989) mencionan que en los representantes de la sect. Americanae, todas las brácteas se entrelazan para formar una vía helicoidal hacia la cavidad del sicono, un arreglo que coincide al encontrado en F. tuerckheimii (Figura 1F). Ramírez (1974) también indicó que las avispas del género Pegoscapus tienen adaptaciones morfológicas que les permiten la entrada a través del ostíolo (e. g. apéndices mandibulares pequeños y con lamelas; Figura 6C). Este tipo de ostíolo es descrito por Machado et al. (2013) en $F$. enormis, especie restringida a Sudamérica, quienes también encontraron, en las brácteas ostiolares externas, los tricomoides, de naturaleza secretora, lo que concuerda con F. tuerckheimii (Figuras 2-4). Se ha encontrado que la interacción Ficus-Agaonidae puede estar vinculada por la emisión de compuestos volátiles del sicono (Grison-Pigé et al. 2002a, b) y es posible que los tricomoides y osmóforos puedan desempeñar un papel relevante al respecto; su comprobación demanda la realización de estudios particulares a futuro. La presencia de peines coxales en las patas delanteras y de las corbículas en la avispa polinizadora de $F$. tuerckheimii (Figura 6E-F) es indicadora de una polinización activa (Kjellberg et al. 2001, Jousselin 

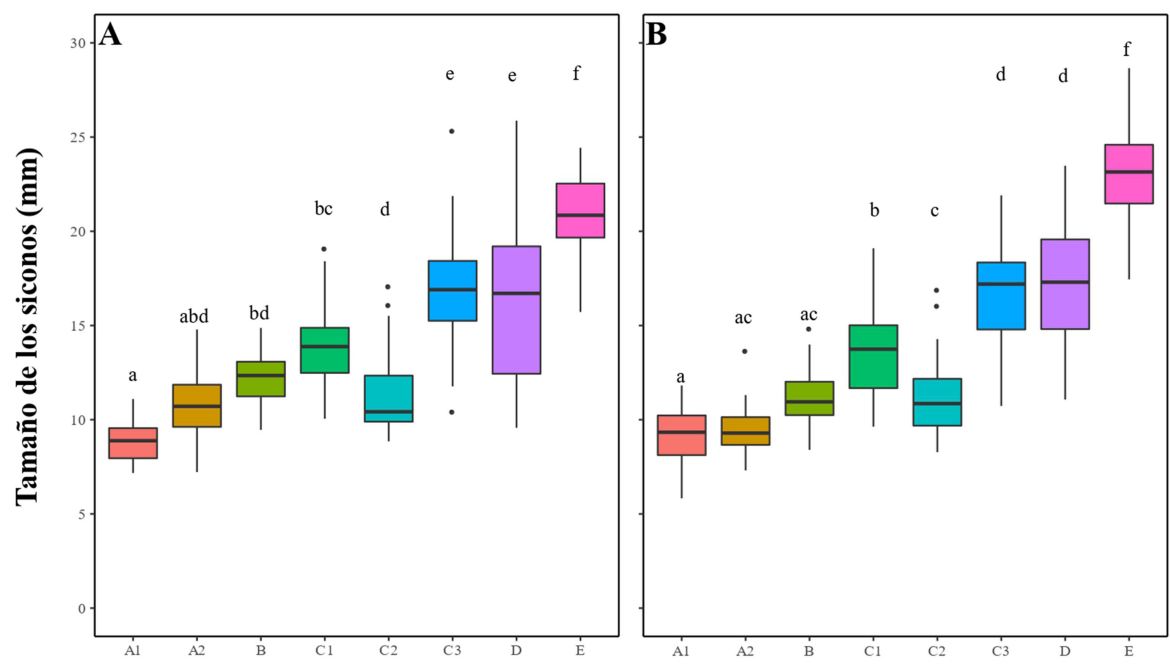

Fases del desarrollo del sicono

Figura 5. Comparaciones múltiples de medias (prueba poshoc de Tukey), para evaluar las diferencias en largo (A) o ancho (B), entre las distintas fases de desarrollo de los siconos de Ficus tuerckheimii.

et al. 2003, Zhang \& Yang, 2017). Este tipo de polinización se asocia con siconos monoicos (sistema reproductivo característico de las especies de la sect. Americanae) y que presentan sinestigma (Verkerke 1989, Jousselin et al. 2003), estructura registrada en el presente estudio (Figura $2 \mathrm{H}, \mathrm{K}$ ).

La Fase D, en F. tuerckheimii, puede ser inferida por la consistencia ligeramente blanda al tacto del sicono y por su coloración primordialmente amarilla (Figura 1H-I). Este color de sicono se ha observado también en otras especies incluidas anteriormente en el complejo $F$. aurea, que son similares en distintos aspectos de su biología reproductiva (Hernández-Esquivel et al. 2020). Uno de ellos es que en $F$. aurea, los orificios para que las avispas hembra salgan del sicono, se ubican en su pared (Hernández-Sosa \& Saralegui-Boza, 2001), evento que también fue observado en la especie bajo estudio (Figura 1I-J). Cabe destacar que, en otras especies, estos orificios pueden ser cavados a través del ostíolo, como acontece en $F$. petiolaris (Piedra-Malagón et al. 2019).

Por otro lado, la Fase E resultó la más contrastante entre las descritas para $F$. tuerckheimii, ya que el sicono alcanza sus mayores dimensiones (Figura 5), además de que se torna rojo y ligeramente dulce, ambas características que comparte con las especies previamente incluidas dentro del complejo $F$. aurea (Ibarra-Manríquez et al. 2012, Hernández-Esquivel et al. 2020). Herre (1996) destaca que entre las especies que estudió en Panamá, del subg. Urostigma (ahora subg. Spherosuke), existe una asociación de siconos pequeños, que maduran de color rojo y que son dispersados por aves, características que coinciden con el síndrome de dispersión ornitócora asociado a cierto grupo de especies de Ficus (Lomáscolo et al. 2008, 2010). Herre
(1996) también asocia estos atributos con una producción de frutos asincrónica, aunque no es claro si se refiere a una asincronía poblacional o la que puede desplegar un individuo. Si bien determinar esta estrategia fenológica no fue un objetivo del presente trabajo, las observaciones de campo realizadas a lo largo del estudio muestran que la asincronía en la copa de un individuo es posible, ya que en una ramilla se pueden registrar siconos en distintas fases de desarrollo, tanto en la localidad de estudio (Figura 1), como en otras regiones de México. Esta estrategia reproductiva también se registró en $F$. petiolaris por Piedra-Malagón et al. (2019). Smith \& Bronstein (1996) indican que la asincronía individual observada en $F$. insipida y $F$. petiolaris puede explicarse por la baja densidad de árboles a nivel regional, para permitir la persistencia de las avispas polinizadoras. Por su parte, Bronstein \& Patel (1992) destacan que la maduración asincrónica o sincrónica de $F$. aurea, dependerá de que existan condiciones adecuadas para el vuelo de los polinizadores. Este tema es sin duda relevante para el entendimiento de la relación mutualista entre $F$. tuerckheimii y las avispas que lo polinizan y sería interesante realizar estudios futuros.

Con respecto a las avispas que se encontraron en los siconos de F. tuerckheimii, Ramírez (1970) destacó que en esta especie existen dos polinizadoras del género Pegoscapus (P. carlosi Ramírez y P. mariae Ramírez). A pesar de que Wiebes (1995) reconoce ambas especies y las incluye dentro de la clave del género, separar las especies con base en la morfología que despliegan no es totalmente confiable, ya que se han detectado especies crípticas (Molbo et al. 2003). Ante estos problemas taxonómicos, el nivel de determinación de las especies de Pegoscapus (avispas 
polinizadoras) e Idarnes (avispas parásitas) en el presente estudio, se limita sólo a nivel del género.

El presente trabajo aporta información para caracterizar el desarrollo del sicono de $F$. tuerckheimii, especialmente el reconocimiento por medio de características externas e internas de las fases críticas (B y D), en las que interactúa con avispas polinizadoras o parásitas. También deja abierta una serie de interrogantes que será interesante resolver a futuro, como la naturaleza anatómica de su disco basal, determinar las especies de polinizadoras y parásitas con las que interactúa en las localidades de estudio, cuantificar el grado de asincronía de los siconos a nivel individual o poblacional, la amplitud de la fauna de frugívoros que dependen de la disponibilidad de siconos en Fase E o si lo encontrado en la presente investigación, es similar en otras localidades de México y Centroamérica. Ante la limitada información que existe para el desarrollo de los siconos para las especies americanas, se espera que el presente estudio motive la realización de investigaciones similares en otras especies y localidades del Neotrópico.
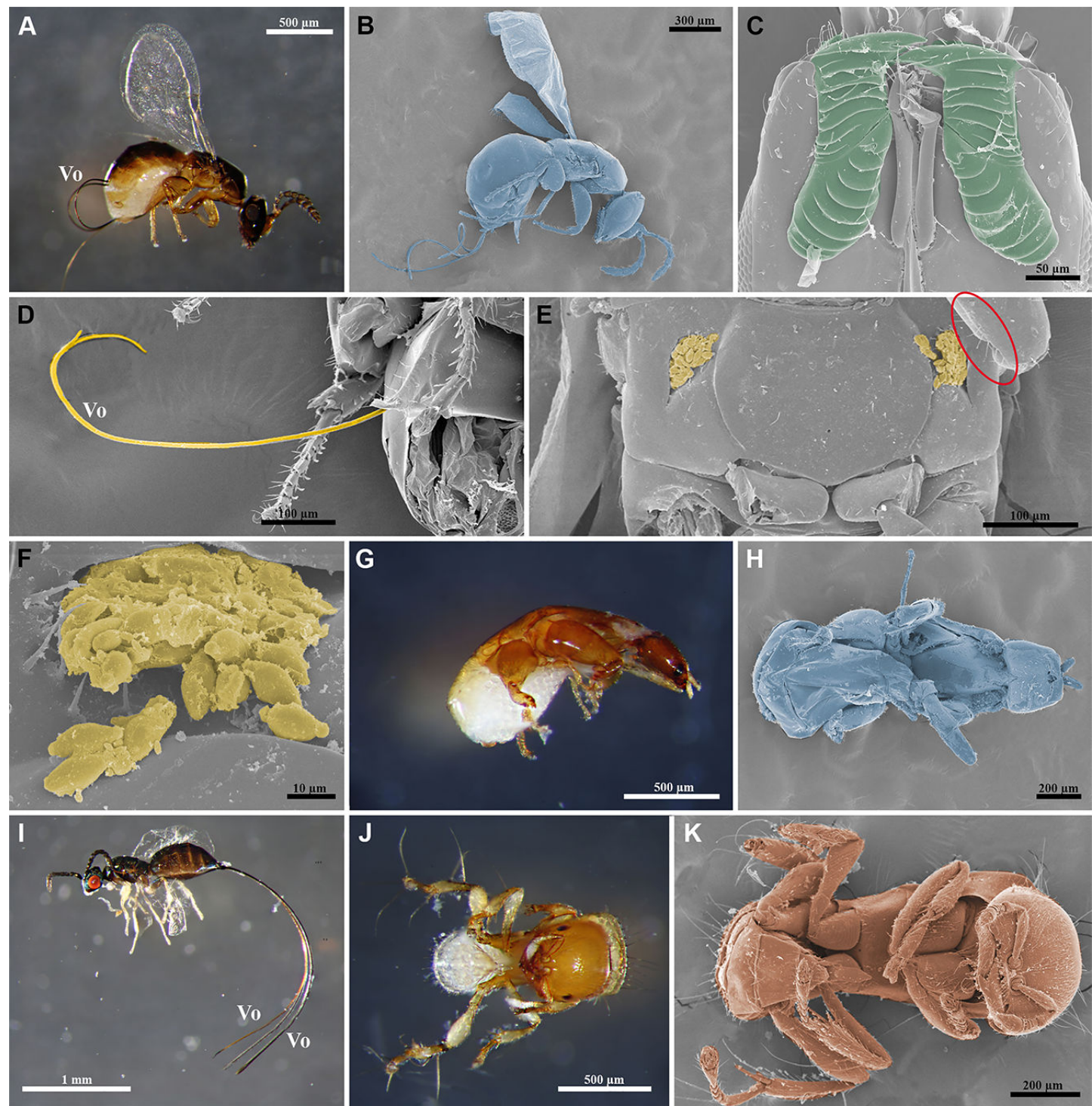

Figura 6. Avispas Agaonidae asociadas al sicono de Ficus tuerckheimii. A-F) Avispa polinizadora hembra (Pegoscapus sp.). A-B) Avispa fotografiada en microscopio estereoscópico y de barrido, respectivamente. C) Mandíbulas con lamelas ventrales (en verde). D) Valvas del ovipositor (en amarillo). E) Corbículas conteniendo granos de polen (en amarillo); peines coxales presentes (elipse roja). F) Detalle de las corbículas con granos de polen (en amarillo). G-H) Avispa macho, áptero, de Pegoscapus sp., fotografiada en microscopio estereoscópico y de barrido, respectivamente. I) Avispa parasitoide (Idarnes sp.), en el que destacan sus ojos rojos y su largo ovipositor. J-K) Avispa macho, áptera, de Idarnes sp., fotografiada en microscopio estereoscópico y de barrido, respectivamente. Vo (valvas del ovipositor). 


\section{Agradecimientos}

Los autores agradecen a la Dra. Eva María Piedra Malagón por su apoyo durante las colectas de campo. A la M. en C. Nadia Castro Cárdenas, por el apoyo en el trabajo de campo y los comentarios a la primera versión del manuscrito. A la Dra. Silvia Espinosa Matías, por el procesamiento y toma de fotomicrografías en el microscopio electrónico de barrido y a la M. en C. Ana Isabel Bieler, por la toma de fotomicrografías en el microscopio óptico. Al M. en C. Pactli Fernando Ortega González y Dr. William Ramírez Benavides, por la ayuda en la identificación de las avispas. El Dr. Armando Navarrete Segueda realizó los análisis estadísticos sobre la variación de las dimensiones del sicono. También agradecemos los atinados comentarios de dos revisores anónimos, los cuales ayudaron a mejorar diversos aspectos de nuestro trabajo. Finalmente, también se agradece al Programa de Apoyo a la Investigación e Innovación Tecnológica (PAPIIT, UNAM, IN208217, Filogeografía comparada del complejo taxonómico Ficus aurea (Moraceae) y sus avispas polinizadoras del género Pegoscapus (Agaonidae), otorgado a Guillermo Ibarra-Manríquez), por el financiamiento económico otorgado al presente estudio y por la beca otorgada a la primera autora para la conclusión de sus estudios de licenciatura.

\section{Literatura citada}

Baijnath H, Ramcharun S. 1983. Aspects of pollination and floral development in Ficus capensis Thunb. (Moraceae). Bothalia 14: 883-888. DOI: https://doi.org/10.4102/abc. v14i3/4.1257

Baijnath H, Naicker S. 1989. Developmental anatomy of Ficus ingens syconia in relation to its wasp faunula. South African Journal of Botany 55: 409-421. DOI: https://doi.org/10.1016/S0254-6299(16)31164-4

Berg CC. 1989. Classification and distribution of Ficus. Experientia 45: 605-611. DOI: https://doi.org/10.1007/ BF01975677

Berg CC. 1990. Reproduction and evolution in Ficus (Moraceae): Traits connected with the adequate rearing of pollinators. Memoirs of the New York Botanical Garden 55: 169-185.

Berg CC. 2007. Proposals for treating four species complexes in Ficus subgenus Urostigma section Americanae (Moraceae). Blumea 52: 295-312. DOI: https://doi.org/10.3767/000651907X609034

Berg CC, Corner EJH. 2005. Moraceae: Ficeae. Flora Malesiana Serie I 17. Leiden: Nationaal Herbarium Nederland. ISBN: 90-71236-61-7 DOI: https://doi.org/ $\underline{10.1017 / \mathrm{S} 0960428607064311}$
Bronstein JL, Patel A. 1992. Causes and consequences of within-tree phenological patterns in the Florida strangling fig, Ficus aurea (Moraceae). American Journal of Botany 79: 41-48. DOI: https://doi.org/ $\underline{10.2307 / 2445195}$

Bronstein JL, Hossaert-McKey M. 1995. Hurricane Andrew and a Florida fig pollination mutualism: resilience of an obligate interaction. Biotropica 27: 373-381. DOI: https://doi.org/10.2307/2388922

Christenhusz MJM, Byng JW. 2016. The number of known plants species in the world and its annual increase. Phytotaxa 261: 201-217. DOI: https://doi.org/10.11646/ phytotaxa.261.3.1

Clement WL, Weiblen GD. 2009. Morphological evolution in the mulberry family (Moraceae). Systematic Botany 34: 530-552. DOI: https://doi.org/10.1600/036364409 $\underline{789271155}$

Cook JM, Rasplus JY. 2003. Mutualists with attitude: coevolving fig wasps and figs. Trends in Ecology and Evolution 18: 241-248. DOI: https://doi.org/10.1016/ S0169-5347(03)00062-4

Datwyler SL, Weiblen GD. 2004. On the origin of the fig: phylogenetic relationships of Moraceae from ndhF sequences. American Journal of Botany 91: 767-777. DOI: https://doi.org/10.3732/ajb.91.5.767

Fan KY, Bain A, Tzeng HY, Chiang YP, Chou LS, KuoHuang LL. 2019. Comparative anatomy of the fig wall (Ficus, Moraceae). Botany 97: 417-426. DOI: https:// doi.org/10.1139/cjb-2018-0192

Galil J, Eisikowitch D. 1968. On the pollination ecology of Ficus sycomorus in East Africa. Ecology 49: 259-269. DOI: https://doi.org/10.2307/1934454

Gibernau M, Hossaert-McKey M, Anstett MC, Kjellberg F. 1996. Consequences of protecting flowers in a fig: a oneway trip for pollinators? Journal of Biogeography 23: 425-432. DOI: https://doi.org/10.1111/j.1365-2699.1996. tb00004.x

Grison-Pigé L, Bessière JM, Hossaert-Mckey M. 2002a. Specific attraction of fig-pollinating wasps: role of volatile compounds released by tropical figs. Journal of Chemical Ecology 28: 283-295. DOI: https://doi.org/ 10.1023/a:1017930023741

Grison-Pigé L, Hossaert-Mckey M, Greeff JM, Bessière JM. 2002b. Fig volatile compounds - a first comparative study. Phytochemistry 61: 61-71. DOI: https://doi.org/ $\underline{\text { 10.1016/s0031-9422(02)00213-3 }}$

Hernández-Esquivel K, Piedra-Malagón EM, CornejoTenorio G, Mendoza-Cuenca L, González-Rodríguez A, Ruiz-Sánchez E, Ibarra-Manríquez G. 2020. Unraveling the extreme morphological variation in the neotropical Ficus aurea complex (subgen. Spherosuke, sect. Americanae, Moraceae). Journal of Systematics and 
Evolution 58: 263-281. DOI: https://doi.org/10.1111/ jse. 12564

Hernández-Sosa A, Saralegui-Boza H. 2001. Contribución al conocimiento de la biología del sicono de Ficus aurea (Moraceae). Revista del Jardín Botánico Nacional 22: 45-48.

Herre EA. 1996. On overview of studies on a community of Panamanian figs. Journal of Biogeography 23: 593-607. DOI: https://doi.org/10.1111/j.1365-2699.1996.tb00020.x

Hothorn T, Bretz F, Westfall P. 2008. Simultaneous Inference in General Parametric Models. Biometrical Journal 50: 346-363. DOI: https://doi.org/10.1002/bimj. $\underline{200810425}$

Ibarra-Manríquez G, Wendt TL. 1992. Ficus, Subgenus Pharmacosycea (Moraceae) in Veracruz, Mexico. Botanical Sciences 52: 3-29. DOI: https://doi.org/10.17 $\underline{129 / \text { botsci. } 1403}$

Ibarra-Manríquez G, Cornejo-Tenorio G, GonzálezCastañeda N, Piedra-Malagón EM, Albino L. 2012. El género Ficus L. (Moraceae) en México. Botanical Sciences 90: 389-452. DOI: https://doi.org/10.17129/ botsci.472

Jousselin E, Rasplus JY, Kjellberg F. 2003. Convergence and coevolution in a mutualism: evidence from a molecular phylogeny of Ficus. Evolution 57: 1255-1269. DOI: https://doi.org/10.1554/02-445

Kjellberg F, Jousselin E, Bronstein JL, Patel A, Yokoyama J, Rasplus JY. 2001. Pollination mode in fig wasps: the predictive power of correlated traits. Proceedings of the Royal Society of London. Series B: Biological Sciences 268: 1113-1121. DOI: https://doi.org/10.1098/rspb.200 $\underline{1.1633}$

Korine C, Kalko EKV, Herre EA. 2000. Fruit characteristics and factors that affect fruit removal in a Panamanian community of strangler figs. Oecologia 123: 560-568. DOI: https://doi.org/10.1007/PL00008861

Lomáscolo SB, Speranza P, Kimball RT. 2008. Correlated evolution of fig size and color supports the dispersal syndromes hypothesis. Oecologia 156: 783-796. DOI: https://doi.org/10.1007/s00442-008-1023-0

Lomáscolo SB, Levey DJ, Kimball RT, Bolker BM, Alborn HT. 2010. Dispersers shape fruit diversity in Ficus (Moraceae). Proceedings of the National Academy of Sciences of the United States of America 107: 14668-14672. DOI: https://doi.org/10.1073/pnas.100877 $\underline{3107}$

Machado AFP, Matos A, Espolador CAE. 2013. Secretory structures at syconia and flowers of Ficus enormis (Moraceae): A specialization at ostiolar bracts and the first report of inflorescence colleters. Flora-Morphology, Distribution, Functional Ecology of Plants 208: 45-51. DOI: https://doi.org/10.1016/j.flora.2012.12.005
Machado C, Robbins N, Gilbert MTP, Herre EA. 2005. Critical review of host specificity and its coevolutionary implications in the fig/fig-wasp mutualism. Proceedings of the National Academy of Sciences of the United States of America 102: 6558-6565. DOI: https://doi.org/10. 1073/pnas.0501840102

Márquez-Guzmán J, Wong JG, Pérez-Pacheco M, López ML, Murguía G, Collazo, M. 2016 Técnicas de Laboratorio para el Estudio del Desarrollo de Angiospermas. Ciudad de México: Universidad Nacional Autónoma de México. ISBN: 978-607-02-8252-2

Molbo D, Machado CA, Sevenster JG, Keller L, Herre EA. 2003. Cryptic species of fig-pollinating wasps: Implications for the evolution of the fig-wasp mutualism, sex allocation, and precision of adaptation. Proceedings of the National Academy of Sciences of the United States of America 100: 5867-5872. DOI: https://doi.org/10.1 $\underline{073 / \text { pnas.0930903100 }}$

Newton LE, Lomo A. 1979. The pollination of Ficus vogelii in Ghana. Botanical Journal of the Linnean Society 78: 21-30. DOI: https://doi.org/10.1111/j.10958339.1979.tb02183.x

Pederneiras LC, Carauta JPP, Romaniuc-Neto S, Mansano VDF. 2015. An overview of the infrageneric nomenclature of Ficus (Moraceae). Taxon 64: 589-594. DOI: https://doi.org/10.12705/643.12

Piedra-Malagón EM, Hernández-Ramos B, MirónMonterrosas A, Cornejo-Tenorio G, Navarrete-Segueda A, Ibarra-Manríquez G. 2019. Syconium development in Ficus petiolaris (Ficus, sect. Americanae, Moraceae) and the relationship with pollinator and parasitic wasps. Botany 97: 190-203. DOI: https://doi.org/10.1139/cjb2018-0095

POWO. 2020. Plants of the World Online. Royal Botanic Gardens, Kew. http://www.plantsoftheworldonline.org (accessed March 30, 2020).

R Core Team. 2018. R: A language end environment for statistical computing. R Foundation for Statistical Computing. Vienna, Austria. http://www.R-project.org/ (accessed March 13, 2020).

Ramírez W. 1970. Host specificity of fig wasps. Evolution 24: 680-691. DOI: https://doi.org/10.1111/j.1558-5646. 1970.tb01804.x

Ramírez W. 1974. Coevolution of Ficus and Agaonidae. Annals of the Missouri Botanical Garden 61: 770-780. DOI: https://doi.org/10.2307/2395028

Shanahan M, So S, Compton SG, Corlett R. 2001. Figeating by vertebrate frugivores: a global review. Biological Reviews 76: 529-572. https://doi.org/10.1017/ $\underline{\text { S1464793101005760 }}$

Smith CM, Bronstein JL. 1996. Site variation in reproductive synchrony in three neotropical figs. Journal of Biogeography 23: 477-486. DOI: https://doi.org/ 10.1111/j.1365-2699.1996.tb00009.x 
Verkerke W. 1986. Anatomy of Ficus ottoniifolia (Moraceae) syconia and the role in the fig-fig wasp simbiosis. Proceedings of the Koninklijke Nederlandse Akademie van Wetenschappen/C 89: 443-469.

Verkerke W. 1987. Syconial anatomy of Ficus asperifolia (Moraceae), a gynodioecious tropical fig. Proceedings of the Koninklijke Nederlandse Akademie van Wetenschappen/C 90: 461-492.

Verkerke W. 1988. Sycone morphology and its influence on the flower structure of Ficus sur (Moraceae). Proceedings of the Koninklijke Nederlandse Akademie van Wetenschappen/C 91: 319-344.

Verkerke W. 1989. Structure and function of the fig. Experientia 45: 612-622. DOI: https://doi.org/10.1007/ BF01975678

Editor de sección: María Silvia Ferrucci

Contribuciones de los autores: GDP realizó el trabajo de campo y laboratorio, participó en la elaboración de los resultados y en la escritura del manuscrito. SVS diseñó el trabajo, apoyó la generación e interpretación de los resultados y participó en la escritura del manuscrito. GCT elaboró las figuras y participó en la escritura del manuscrito. GIM colaboró en el diseñó el trabajo y participó en la escritura del manuscrito. Todos los autores revisaron la versión final del trabajo.
Weiblen GD. 2002. How to be a fig wasp. Annual Review of Entomology 47: 299-330. DOI: https://doi.org/10.1146/ annurev.ento.47.091201.145213

Wiebes JT. 1995. The New World Agaoninae (pollinators of Ficus). Amsterdam: Koninklijke Nederlandse Akademie van Wetenschappen. ISBN: 0-444-85798-2

Zhang G, Song Q, Yang D. 2006. Phenology of Ficus racemosa in Xishuangbanna, Southwest China. Biotropica 38: 334-341. DOI: https://doi.org/10.1111/ j.1744-7429.2006.00150.x

Zhang X, Yang D. 2017. Occurrence of internally ovipositing non-agaonid wasps and pollination mode of the associated agaonid wasps. Plant Diversity 39: 130-134. DOI: https://doi.org/10.1016/j.pld.2017.04.002 